Pacific

Journal of

Mathematics

STABILITY OF THE CHENG-YAU GRADIENT ESTIMATE

QI S. ZHANG

Volume $225 \quad$ No. 2

June 2006 


\title{
STABILITY OF THE CHENG-YAU GRADIENT ESTIMATE
}

\author{
QI S. ZHANG
}

\begin{abstract}
We prove that the Cheng-Yau gradient estimate on positive harmonic functions on manifolds with nonnegative Ricci curvature is globally stable under certain perturbations of the metric. In some cases, one only needs the condition $\operatorname{Ricci}(x) \geq-\varepsilon /\left(1+d(x)^{2+\delta}\right)$, with $\delta>0$ and $\varepsilon>0$ sufficiently small.
\end{abstract}

\section{Introduction}

One of the most useful inequalities in geometric analysis is the Cheng-Yau estimate on the gradient of positive harmonic functions.

Theorem (Cheng-Yau). Let $M$ be a complete manifold of dimension $n \geq 2$ and Ricci curvature bounded below by $-k$, with $k \geq 0$. Suppose $u$ is any positive harmonic function in a geodesic ball $B\left(x_{0}, r\right) \subset M$. Then

$$
\sup _{B\left(x_{0}, r / 2\right)} \frac{|\nabla u|}{u} \leq \frac{c_{n}}{r}+c_{n} \sqrt{k}
$$

where $c_{n}$ depends only on the dimension $n$.

When $k=0$ (so $M$ has nonnegative Ricci curvature), the Cheng-Yau estimate becomes

$$
\sup _{B\left(x_{0}, r / 2\right)} \frac{|\nabla u|}{u} \leq \frac{c_{n}}{r}
$$

which is sharp, as we can see by considering the Euclidean case. However, even if $M$ contains a small compact region where the Ricci curvature is not nonnegative, estimate (1-1) becomes very different from (1-2) when $r$ is large, because of the $\sqrt{k}$ term. Whether estimate (1-2) is stable under perturbation has been an open question for some time, in light of the known stability results on weaker properties of harmonic functions, such as the Harnack inequality.

Here we confirm that (1-2) is stable when the nonpositive part of the Ricci curvature is sufficiently small in an integral sense.

MSC2000: 58J05, 58J35.

Keywords: harmonic functions, log gradient bound, stablility. 
Some smallness condition for the nonpositive part of the Ricci curvature is surely necessary for (1-2) to hold. For instance, if the nonpositive part of the Ricci curvature is so large that $M$ admits a bounded nonconstant harmonic function, clearly (1-2) cannot hold.

Throughout the paper $\Delta$ is the Laplace-Beltrami operator, $d(x, y)$ is the distance between $x$ and $y$, and $d(x)$ is the distance between $x$ and a fixed reference point. $|B(x, r)|$ denotes the volume of the geodesic ball of radius $r$ centered at $x$.

We now lay out the basic assumptions required. As will be explained later, these assumptions are stable under certain perturbations of the metric.

Assumption A. $M$ is a complete noncompact Riemannian manifold of dimension $n \geq 2$ satisfying the volume doubling property

$$
|B(x, 2 r)| \leq 2^{v}|B(x, r)|
$$

for all $x \in M, r>0$ and some $v>0$.

Assumption B. The heat kernel $G$ of the Laplace-Beltrami operator satisfies a Gaussian upper bound

$$
G(x, t ; y, 0) \leq \frac{B_{1}}{|B(x, \sqrt{t})|} \exp \frac{-b_{1} d^{2}(x, y)}{t},
$$

for some $b_{1}, B_{1}>0$, and all $x, y \in M$ and $t>0$.

Several conditions are known to be equivalent to assumptions A and B. For instance, it was proved in [Grigoryan 1991] that the two assumptions together are equivalent to:

Faber-Krahn inequality. For any $x \in M, r>0$, and nonempty subset $\Omega \subset B(x, r)$,

$$
\lambda_{1}(\Omega) \geq \frac{c}{r^{2}}\left(\frac{|B(x, r)|}{|\Omega|}\right)^{2 / v} .
$$

Here $\lambda_{1}(\Omega)$ is the first Dirichlet eigenvalue of $\Omega$ and $c>0$.

It is also known [Saloff-Coste 1992] that if Assumption A is satisfied, Assumption $\mathrm{B}$ is equivalent to:

Assumption B'. The following Sobolev inequality holds for all $\phi \in C_{0}^{\infty}(B(x, r))$, $x \in M, r>0$, and a fixed $\alpha>2$ :

$$
\left(\int \phi^{2 \alpha /(\alpha-2)} d y\right)^{(\alpha-2) / \alpha} \leq S_{0}|B(x, r)|^{-2 / \alpha} \int\left(r^{2}|\nabla \phi|^{2}+\phi^{2}\right) d y .
$$


Also under Assumption A, it is known that Assumption B is implied by a certain Poicaré inequality [Saloff-Coste 1992; Grigoryan 1991], and by a certain meanvalue inequality [Li and Wang 1999]. There is an extensive literature on manifolds satisfying various global conditions including the ones mentioned above; see [Grigor'yan 1999; Saloff-Coste 2002] and references therein.

There exist many manifolds satisfying assumptions Assumption A and Assumption $\mathrm{B}$, among them is the class of manifolds quasiisometric to manifolds with nonnegative Ricci curvature, and connected sums of two copies of $\mathbb{R}^{n}$. See [Coulhon and Saloff-Coste 1995], for example.

Next we introduce the conditions on the nonpositive part of the Ricci curvature that will imply the global Cheng-Yau estimate (1-2). The conditions, in general integral form first, will be elucidated in the Corollary below by simple conditions. Essentially, the nonpositive part of the Ricci curvature is required to be small and decay sufficiently fast near infinity.

Let $\lambda=\lambda(x)$ be the lowest eigenvalue of $\operatorname{Ric}(x)$, for $x \in M$. We use the notation

$$
V(x):=\frac{1}{2}(|\lambda(x)|-\lambda(x))
$$

Assumption C. The function $V$ lies in $L^{\infty}(M)$ and there exist $\varepsilon_{0}>0$ and $K>0$ such that

$$
N(V) \equiv \sup _{x \in M} \int_{0}^{\infty} \int_{M} \frac{e^{-d(x, y)^{2} / t}}{|B(x, \sqrt{t})|} V(y) d y d t<\varepsilon_{0}
$$

and

$$
\int_{M} V(y) \phi^{2}(y) d y<\frac{1}{11 n} \int_{M}|\nabla \phi(x)|^{2} d x+\frac{K}{r^{2}} \int_{M} \phi^{2}(y) d y
$$

for all $\phi \in C_{0}^{\infty}(B(x, r))$.

Here is the key result in this paper.

Main Theorem. Suppose $M$ satisfies Assumptions $A$ and $B$. There exists $\varepsilon_{0}>0$, depending only on the parameters in those assumptions, so that if Assumption $C$ on the Ricci curvature holds, the following statement holds.

Let $u$ be a positive harmonic function in the ball $B(x, r)$. Then

$$
\sup _{y \in B(x, r / 2)} \frac{|\nabla u(y)|}{u(y)} \leq \frac{C_{0}}{r}
$$

where $C_{0}$ depends only on the assumptions' parameters $v, b_{1}, B_{1}, \varepsilon_{0}, K$ and $n$.

Remark 1. In many cases, the condition on $V$ in the theorem simply means that

$$
\operatorname{Ric}(x) \geq-\frac{\varepsilon}{1+d(x)^{2+\delta}}
$$

for some small enough $\varepsilon>0$ and $\delta>0$. This is indicated in the Corollary below. In general, the first equation in Assumption $\mathrm{C}$ is a Kato-type condition and the 
second condition takes the form of Hardy's inequality; that is, for $f \in C_{0}^{\infty}\left(\mathbb{R}^{n}\right)$ $(n \geq 3)$, we have

$$
\frac{(n-2)^{2}}{4} \int_{\mathbb{R}^{n}} \frac{1}{|x|^{2}} f(x)^{2} d x \leq \int_{\mathbb{R}^{n}}|\nabla f|^{2}(x) d x .
$$

For versions of Hardy's inequality in manifolds, see [Carron 1997; Ancona 1990]. In many situations the first item implies the second, as we now see:

Corollary. Suppose that $M$ satisfies the Sobolev inequality Assumption $B^{\prime}$ with $\alpha=n$ and that $|B(x, r)|$ is comparable with $r^{n}$, for $n>2$, meaning that there exists $a>0$ such that $a^{-1} r^{n} \leq|B(x, r)| \leq a r^{n}$ for all $x \in M$ and $r>0$. Then the gradient bound (1-2) holds if

$$
\operatorname{Ric}(x) \geq-\frac{\varepsilon}{1+d(x)^{2+\delta}}
$$

for a sufficiently small $\varepsilon>0$ and $\delta>0$. Here $\varepsilon$ depends only on $b_{1}, B_{1}, \delta, a, n$.

In particular, if $M$ is a small compact perturbation of $\mathbb{R}^{n}$, where $n \geq 3$, Equation (1-2) holds.

Likewise, (1-2) holds if $M$ is a small compact perturbation of a manifold of dimension at least 3 that has nonnegative Ricci curvature and maximum volume growth (that is, $\left.|B(x, r)| \sim r^{n}\right)$.

Proof. Since $M$ satisfies the extra conditions in the volume of geodesic balls in the corollary, it is easy to see that

$$
N(V)=\sup _{x \in M} \int_{0}^{\infty} \int_{M} \frac{e^{-d(x, y)^{2} / t}}{|B(x, \sqrt{t})|} V(y) d y d t \leq c \sup _{x \in M} \int_{M} \frac{d(x, y)^{2}}{|B(x, d(x, y))|} V(y) d y .
$$

Write

$$
K(V) \equiv \sup _{x \in M} \int_{M} \frac{d(x, y)^{2}}{|B(x, d(x, y))|} V(y) d y
$$

By direct calculation we see that $V(x) \leq \varepsilon /\left(1+d(x)^{2+\delta}\right)$ implies

$$
\begin{aligned}
K(V) & \leq \sup _{x \in M} \int_{d(x, y) \geq d(y) / 2} \frac{d(x, y)^{2}}{|B(x, d(x, y))|} V(y) d y \\
& \quad+\sup _{x \in M} \int_{d(x, y) \leq d(y) / 2} \frac{d(x, y)^{2}}{|B(x, d(x, y))|} V(y) d y \\
& \leq C \varepsilon .
\end{aligned}
$$

Next, given $\phi \in C_{0}^{\infty}\left(B\left(x_{0}, r\right)\right)$,

$$
\int_{M} V(x) \phi^{2}(x) d x \leq\left(\int_{M} V^{n / 2}(x) d x\right)^{2 / n}\left(\int_{M} \phi^{2 n /(n-2)}(x) d x\right)^{(n-2) / n} .
$$


By the Sobolev inequality,

$\int_{M} V(x) \phi^{2}(x) d x \leq S_{0}\left(\int_{M} V^{n / 2}(x) d x\right)^{2 / n}\left(\int_{M}|\nabla \phi|^{2}(x) d x+\frac{k}{r^{2}} \int_{M} \phi^{2}(x) d x\right)$.

A simple calculation then shows that

$$
\int_{M} V(x) \phi^{2}(x) d x \leq C \varepsilon S_{0}\left(\int_{M}|\nabla \phi|^{2}(x) d x+\frac{k}{r^{2}} \int_{M} \phi^{2}(x) d x\right) .
$$

Hence all the conditions of the Main Theorem are satisfied when $\varepsilon$ is sufficiently small.

There are plenty of examples of such manifolds due the stability of $G(x, t ; y, 0)$ under perturbation of the metric. For instance, let $M$ be $\mathbb{R}^{n}$ equipped with a metric coming from a small perturbation of the Euclidean metric. Here $n \geq 3$. Then by standard results $B_{1}$ and $b_{1}$ can be chosen to be close to $1 /(2 \sqrt{\pi})^{n}$ and $1 / 4$, the Euclidean constants. At the same time, the nonpositive part of the Ricci curvature can be arbitrarily small. Therefore the above quantity $N(V)$ can be arbitrarily small while $\varepsilon_{0}$, depending only on $B_{1}, b_{1}$ and the doubling constant, is bounded away from zero. Thus $N(V)<\varepsilon_{0}$.

The last statement in the corollary is proved in the same manner.

Remark 2. The constant $\varepsilon_{0}$ and $C_{0}$ in the Main Theorem can be estimated explicitly, as indicated in the proof. The assumption $V \in L^{\infty}(M)$ is not necessary. But we will not seek the full generality.

It is not clear whether the current method can show the Li-Yau gradient estimate on caloric functions [1986] is stable.

\section{Proof of the Main Theorem}

Let $u$ be a positive solution of $\Delta u=0$. The proof is carried out in several steps.

2.1. Derivation of an equation for $\log \boldsymbol{u}$. In this initial step we follow [Yau 1975] and [Cheng and Yau 1975]. Set $f=\log u$; then $\Delta f=-|\nabla f|^{2}$. Define

$$
F \equiv|\nabla f|^{2} .
$$

By Bochner's identity, $F$ obeys

$$
\Delta F \geq-2 \nabla f \nabla F+\frac{2}{n} F^{2}-2 V F .
$$

Here $V=V(x)$ is the absolute value of the negative part of the lowest eigenvalue of $\operatorname{Ric}(x)$. 
2.2. Preparing the ground for a bound on $\boldsymbol{F}$. From here on our proof is different from those in [Yau 1975] and [Cheng and Yau 1975], where the maximum principle was used. We will use certain integral estimates motivated by the De Giorgi-NashMoser theory on linear elliptic and parabolic equations.

The idea is to convert (2-1) into a linear inequality and prove that the heat kernel of the corresponding operator satisfies a global Gaussian upper bound when $N(V)$, defined in Assumption $\mathrm{C}$, is sufficiently small. Then one can use the local representation formula for solutions and the Hölder inequality to derive an $L^{\infty}$ bound for $F$. However, it is not clear that the linear operator

$$
-\Delta-2 \nabla f \nabla+\frac{2}{n} F-2 V
$$

associated with (2-1) is positive definite. Hence there is no hope of proving a global Gaussian upper bound for the corresponding heat kernel.

To overcome the difficulty, we consider the function

$$
w=F^{m}, \quad \text { where } m=5 n .
$$

By direct calculation, one easily finds using (2-1) that

$$
\Delta w+2 \nabla f \nabla w-10 F w+10 n V w \geq 0 .
$$

We define the operators $L_{1}$ and $L_{2}$ by

$$
\begin{aligned}
& L_{1}=\Delta+2 \nabla f \nabla-10 F, \\
& L_{2}=\Delta+2 \nabla f \nabla-10 F+10 n V,
\end{aligned}
$$

and their corresponding heat kernels by $G_{1}$ and $G_{2}$. We will eventually prove that $G_{2}$ has a global Gaussian upper bound when $N(V)$ is small. More importantly, the coefficients in the Gaussian upper bound should be independent of $f$, which is not a fixed function. This is achieved by exploiting the special structure of the operator $L_{1}$.

First, we have to show that $G_{1}$ satisfies a global Gaussian upper bound. This is the longest part of the proof, and we do it in the next two sections.

2.3. A mean-value inequality. Set

$$
L_{3}=\Delta+2 \nabla f \nabla-8 F .
$$

We will prove an $L^{2}$ mean-value inequality for positive solutions of

$$
L_{3} w-w_{t} \geq 0 \text {. }
$$


Let $Q_{r}(x, t)$ (or simply $Q_{r}$ ) denote $B(x, r) \times\left[t-r^{2}, t\right]$. The inequality to be proved is

$$
\sup _{Q_{r / 2}(x, t)} w^{2} \leq \frac{C_{7}}{B(x, r) r^{2}}\|w\|_{L^{2}\left(Q_{r}(x, t)\right)}^{2}
$$

Notice that the order-zero term in $L_{3}$ is $-8 F w$ instead of $-10 F w$ in $L_{1}$. This makes $L_{3}$ a "bigger" operator than $L_{1}$.

Fix $\sigma>1$. Let $\eta: \mathbb{R} \rightarrow \mathbb{R}$ be a cutoff function supported in $\left(t-(\sigma r)^{2}, t\right)$ and such that $\eta(s)=1$ for $s \in\left[t-r^{2}, t\right]$ and $\left|\eta^{\prime}\right| \leq 2 /((\sigma-1) r)^{2}$ for $0 \leq \eta \leq 1$. Similarly, let $\phi: \mathbb{R}^{n} \rightarrow[0,1]$ be a function supported in $B(x, \sigma r)$ and such that $\phi(y)=1$ for $y \in B(x, r)$ and

$$
|\nabla \phi| \leq \frac{A}{(\sigma-1) r}, \quad \text { with } A>0 .
$$

Then set $\psi(y, s)=\phi(y) \eta(s)$. Using $w \psi^{2}$ as a test function in (2-3), one obtains

$$
\int_{Q_{\sigma r}}\left(\Delta w-2 \nabla f \nabla w-8 F w-\partial_{s} w\right) w \psi^{2} d y d s \geq 0 .
$$

Integrating by parts, one deduces

$$
\begin{array}{rl}
\int_{Q_{\sigma r}} \nabla\left(w \psi^{2}\right) \nabla w d y d s \leq \int_{Q_{\sigma r}} & 2 \nabla f \nabla w\left(w \psi^{2}\right) d y d s \\
& -\int_{Q_{\sigma r}} 8 F w^{2} d y d s-\int_{Q_{\sigma r}}\left(\partial_{s} w\right) w \psi^{2} d y d s .
\end{array}
$$

By direct calculation,

$$
\begin{aligned}
\int_{Q_{\sigma r}} \nabla\left(w \psi^{2}\right) \nabla w d y d s & =\int_{Q_{\sigma r}} \nabla((w \psi) \psi) \nabla w d y d s \\
& =\int_{Q_{\sigma r}}(\nabla(w \psi)(\nabla(w \psi)-(\nabla \psi) w)+w \psi \nabla \psi \nabla w) d y d s \\
& =\int_{Q_{\sigma r}}\left(|\nabla(w \psi)|^{2}-|\nabla \psi|^{2} w^{2}\right) d y d s .
\end{aligned}
$$

Substituting this in (2-5), we obtain

$$
\begin{aligned}
& \int_{Q_{\sigma r}}|\nabla(w \psi)|^{2} d y d s \\
& \leq \int_{Q_{\sigma r}} 2 \nabla f \nabla w\left(w \psi^{2}\right) d y d s-\int_{Q_{\sigma r}} 8 F w^{2} d y d s \\
& \quad-\int_{Q_{\sigma r}}\left(\partial_{s} w\right) w \psi^{2} d y d s+\int_{Q_{\sigma r}}|\nabla \psi|^{2} w^{2} d y d s
\end{aligned}
$$


Next, notice that

$$
\begin{aligned}
\int_{Q_{\sigma r}}\left(\partial_{s} w\right) w \psi^{2} d y d s & =\frac{1}{2} \int_{Q_{\sigma r}}\left(\partial_{s} w^{2}\right) \psi^{2} d y d s \\
& =-\int_{Q_{\sigma r}} w^{2} \phi^{2} \eta \partial_{s} \eta d y d s+\frac{1}{2} \int_{B(x, \sigma r)} w^{2}(y, t) \phi^{2}(y) d y .
\end{aligned}
$$

Combining this with (2-6), we see that

$$
\begin{aligned}
\int_{Q_{\sigma r}}|\nabla(w \psi)|^{2} d y d s+\frac{1}{2} \int_{B(x, \sigma r)} w^{2}(y, t) \phi^{2}(y) d y & \\
& \leq \int_{Q_{\sigma r}}\left(|\nabla \psi|^{2}+\eta \partial_{s} \eta\right) w^{2} d y d s+\int_{Q_{\sigma r}} 2 \nabla f \nabla w\left(w \psi^{2}\right) d y d s \\
&
\end{aligned}
$$

The first term on the right in (2-7) is already in good shape. We estimate the second as follows:

$$
\begin{array}{rl}
\int_{Q_{\sigma r}} & 2 \nabla f(\nabla w)\left(w \psi^{2}\right) d y d s \\
= & 2 \int_{Q_{\sigma r}} \nabla f(\nabla(w \psi)-w \nabla \psi) w \psi d y d s \\
= & 2 \int_{Q_{\sigma r}} \nabla f \nabla(w \psi) w \psi d y d s-2 \int_{Q_{\sigma r}}(w \psi \nabla f) w \nabla \psi d y d s \\
\leq & \frac{1}{2} \int_{Q_{\sigma r}}|\nabla(w \psi)|^{2} d y d s+4 \int_{Q_{\sigma r}}|\nabla f|^{2}(w \psi)^{2} d y d s \\
& +\int_{Q_{\sigma r}}|\nabla f|^{2}(w \psi)^{2} d y d s+\int_{Q_{\sigma r}} w^{2}|\nabla \psi|^{2} d y d s .
\end{array}
$$

Recall that $|\nabla f|^{2}=F$. Hence this inequality becomes

$$
\begin{aligned}
\int_{Q_{\sigma r}} 2 \nabla f & (\nabla w)\left(w \psi^{2}\right) d y d s \\
\leq & \frac{1}{2} \int_{Q_{\sigma r}}|\nabla(w \psi)|^{2} d y d s+5 \int_{Q_{\sigma r}} F(w \psi)^{2} d y d s+\int_{Q_{\sigma r}} w^{2}|\nabla \psi|^{2} d y d s .
\end{aligned}
$$

Substituting this on the right-hand side of (2-7), we deduce that

$$
\begin{aligned}
\int_{Q_{\sigma r}}|\nabla(w \psi)|^{2} d y d s+\frac{1}{2} \int_{B(x, \sigma r)} w^{2}(y, t) \phi^{2}(y) d y & \\
& \leq 2 \int_{Q_{\sigma r}}\left(2|\nabla \psi|^{2}+\eta \partial_{s} \eta\right) w^{2} d y d s .
\end{aligned}
$$


Thus the terms containing $F$ drop out. For later use we remark that if $w$ satisfies $L_{3} w-w_{t}+h \geq 0$ for some function $h$, we obtain, using exactly the same calculation,

$$
\begin{aligned}
\int_{Q_{\sigma r}}|\nabla(w \psi)|^{2} d y d s & +\frac{1}{2} \int_{B(x, \sigma r)} w^{2}(y, t) \phi^{2}(y) d y \\
& \leq 2 \int_{Q_{\sigma r}}\left(2|\nabla \psi|^{2}+\eta \partial_{s} \eta\right) w^{2} d y d s+\int_{Q_{\sigma r}} h w \psi^{2} d y d s .
\end{aligned}
$$

By direct calculation it is easy to see that, for any $p>1$,

$$
\begin{aligned}
L_{3} w^{p} & =\Delta w^{p}+2 \nabla f \nabla w^{p}-8 F w^{p}-\left(w^{p}\right)_{t} \\
& \geq p(p-1)|\nabla w|^{2} w^{p-2}+8 F(p-1) w^{p} \geq 0 .
\end{aligned}
$$

Hence, by repeating the argument above, we obtain, for any $p>1$,

$$
\begin{aligned}
& \int_{Q_{\sigma r}}\left|\nabla\left(w^{p} \psi\right)\right|^{2} d y d s+\frac{1}{2} \int_{B(x, \sigma r)}\left(w^{p}(y, t)\right)^{2} \phi^{2}(y) d y \\
& \leq 2 \int_{Q_{\sigma r}}\left(2|\nabla \psi|^{2}+\eta \partial_{s} \eta\right)\left(w^{p}\right)^{2} d y d s .
\end{aligned}
$$

Therefore

$$
\begin{aligned}
\int_{Q_{\sigma r}}\left|\nabla\left(w^{p} \psi\right)\right|^{2} d y d s+\frac{1}{2} \int_{B(x, \sigma r)}\left(w^{p}(y, t)\right)^{2} \phi^{2}(y) d y & \\
\leq & \frac{C}{r^{2}} \int_{Q_{\sigma r}}\left(w^{p}\right)^{2} d y d s .
\end{aligned}
$$

Equation (2-9) and the Sobolev inequality lead to the following mean-value inequality via Moser iteration:

$$
\sup _{Q_{r}} w^{2} \leq \frac{B}{\left|Q_{r}\right|} \int_{Q_{2 r}} w^{2} d y d s .
$$

By keeping track of the constants in the computation, we can show that the constant $B$ is independent of $f$ or $F$. For the sake of completeness we give a sketch of the proof. Hölder's inequality,

$$
\iint\left(w^{p} \psi\right)^{2(1+(2 / \alpha))} d y d s \leq \int\left(\int\left(w^{p} \psi\right)^{2 \alpha /(\alpha-2)} d y\right)^{(\alpha-2) / \alpha}\left(\int\left(w^{p} \psi\right)^{2}\right)^{2 / \alpha} d s .
$$

Using the Sobolev inequality of Assumption $\mathrm{B}^{\prime}$, one obtains

$$
\begin{aligned}
& \iint\left(w^{p} \psi\right)^{2(1+(2 / \alpha))} d y d s \leq \\
& S_{0}|B(x, r)|^{-2 / \alpha} \sup _{s \in\left[t-\sigma r^{2}, t\right]}\left(\int\left(w^{p} \psi\right)^{2} d y\right)^{2 / \alpha} \iint\left(r^{2}\left|\nabla\left(w^{p} \psi\right)\right|^{2}+\left(w^{p} \psi\right)^{2} d y d s\right) .
\end{aligned}
$$


This, together with (2-9), implies that

$$
\int_{Q_{\sigma^{\prime} r}(x, t)} w^{2 p \theta} \leq\left(C_{5} S_{0} \lambda(r)^{-1} \int_{Q_{\sigma r}(x, t)} w^{2 p}\right)^{\theta}
$$

where $\theta=1+2 / \alpha, \tau=\sigma-\sigma^{\prime}$ and

$$
\lambda(r)=|B(x, r)|^{2 /(2+\alpha)}(r \tau)^{4 /(2+\alpha)} .
$$

We now set

$$
\tau_{i}=2^{-i-1}, \quad \sigma_{0}=1, \quad \sigma_{i}=\sigma_{i-1}-\tau_{i}=1-\Sigma_{1}^{i} \tau_{j}, \quad p=\theta^{i}
$$

Inequality (2-11) then yields

$$
\int_{Q_{\sigma_{i+1}}(x, t)} w^{2 \theta^{i+1}} \leq C\left(C_{6}^{i+1} \lambda(r)^{-1} \int_{Q_{\sigma_{i} r}(x, t)} w^{2 \theta^{i}}\right)^{\theta}
$$

After iteration, this implies

$$
\left(\int_{Q_{\sigma_{i+1}}(x, t)} w^{2 \theta^{i+1}}\right)^{\theta^{-i-1}} \leq C^{\Sigma \theta^{-j-1}} C_{6}^{-\Sigma(j+1) \theta^{-j-1}}\left(\lambda(r)^{-1}\right)^{\Sigma \theta^{-j}} \int_{Q_{r}(x, t)} w^{2}
$$

where $j$ ranges from 0 to $i$. Letting $i$ go to $\infty$ and noticing that $\Sigma_{j=0}^{\infty} \theta^{-j}=$ $(\alpha+2) / 2$, we arrive at

$$
\sup _{Q_{r / 2}(x, t)} w^{2} \leq \frac{C_{7}}{B(x, r) r^{2}}\|w\|_{L^{2}\left(Q_{r}(x, t)\right)}^{2}
$$

This proves the mean-value inequality for $w$ satisfying $L_{3} w-w_{t} \geq 0$.

2.4. A Gaussian upper bound for $\boldsymbol{G}_{1}$. The proof of the upper bound is done by modifying the standard method due to E. B. Davies [1989]. To prove a bound that is independent of $f$ or $F$, we have to use the special structure of the operator $L_{1}$. For a fixed $\lambda \in \mathbb{R}$ and a fixed bounded function $\psi$ such that $|\nabla \psi| \leq 1$, we write

$$
q(y)=e^{\lambda \psi(y)} \int G_{1}(y, s ; z, 0) e^{-\lambda \psi(z)} h(z) d z,
$$


where $h$ is a smooth compactly supported function. Then

$$
\begin{aligned}
\partial_{s}\|q\|_{2}^{2} & =2 \int_{M} q(y, s) \partial_{s} q(y, s) \\
& =2 \int_{M} e^{\lambda \psi(y)} q(y, s) \int_{M} \partial_{s} G_{1}(y, s ; z, 0) e^{-\lambda \psi(z)} h(z) d z d y \\
& =2 \int_{M} e^{\lambda \psi(y)} q(y, s) \int_{M}\left(\Delta_{y} G_{1}+2 \nabla_{y} f \nabla_{y} G_{1}-10 F G_{1}\right) \\
& =2 I_{1}+4 I_{2}-20 I_{3},
\end{aligned}
$$

where we have set

$$
\begin{aligned}
& I_{1}=\int_{M} \int_{M} e^{\lambda \psi(y)} q(y, s) \Delta_{y} G_{1} e^{-\lambda \psi(z)} h(z) d z d y, \\
& I_{2}=\int_{M} \int_{M} e^{\lambda \psi(y)} q(y, s) \nabla_{y} f \nabla_{y} G_{1} e^{-\lambda \psi(z)} h(z) d z d y, \\
& I_{3}=\int_{M} \int_{M} e^{\lambda \psi(y)} q(y, s) F G_{1} e^{-\lambda \psi(z)} h(z) d z d y .
\end{aligned}
$$

Integrating by parts and employing standard arguments, we get

$$
I_{1} \leq-\int_{M}|\nabla q(y, s)|^{2} d y+c \lambda^{2} \int_{M} q^{2}(y, s) d y .
$$

Next observe that

$$
\begin{aligned}
I_{2} & =\int_{M} e^{\lambda \psi(y)} q(y, s) \nabla_{y} f \int_{M} \nabla_{y} G_{1} e^{-\lambda \psi(z)} h(z) d z d y \\
& =\int_{M} e^{\lambda \psi(y)} q(y, s) \nabla_{y} f \nabla_{y} \int_{M} G_{1}(y, s ; z, 0) e^{-\lambda \psi(z)} h(z) d z d y \\
& =\int_{M} e^{\lambda \psi(y)} q(y, s) \nabla_{y} f \nabla_{y}\left(e^{-\lambda \psi(y)} q(y, s)\right) d y \\
& =\frac{1}{2} \int_{M} e^{2 \lambda \psi(y)} \nabla_{y} f \nabla_{y}\left(\left(e^{-\lambda \psi(y)} q(y, s)\right)^{2}\right) d y \\
& =-\lambda \int_{M} q^{2}(y, s) \nabla_{y} \psi \nabla_{y} f d y-\frac{1}{2} \int_{M} q^{2}(y, s) \Delta_{y} f d y .
\end{aligned}
$$

Since $|\nabla \psi| \leq 1$ and $\Delta_{y} f=-|\nabla f|^{2}=-F$, it follows that

$$
I_{2} \leq \frac{\lambda^{2}}{2} \int_{M} q^{2}(y, s) d y+\frac{1}{2} \int_{M} q^{2}(y, s)\left|\nabla_{y} f\right|^{2} d y+\frac{1}{2} \int_{M} q^{2}(y, s)\left|\Delta_{y} f\right| d y ;
$$


that is,

$$
I_{2} \leq \frac{\lambda^{2}}{2} \int_{M} q^{2}(y, s) d y+\int_{M} q^{2}(y, s) F d y .
$$

Notice also that

$$
I_{3}=\int_{M} q^{2}(y, s) F d y .
$$

Substituting (2-13) and the last two relations in (2-12), we see that the terms containing $F$ are negative. Hence

$$
\partial_{s}\|q(\cdot, s)\|_{2}^{2} \leq c_{0} \lambda^{2}\|q(\cdot, s)\|_{2}^{2},
$$

which implies

$$
\|q(\cdot, s)\|_{2}^{2} \leq e^{c_{0} \lambda^{2} s}\|h\|_{2}^{2}
$$

Now consider the function

$$
u(y, s)=e^{-\lambda \psi(y)} q(y, s),
$$

which is a solution to $L_{1} u-u_{s}=0$ in $M \times(0, \infty)$. Hence

$$
L_{3} u-u_{s}=L_{1} u-u_{s}+2 F u \geq 0,
$$

where $L_{3}$ is defined at the start of Section 2.3. By the mean-value inequality of (2-10) with $Q_{\sqrt{t} / 2}(x, t)=B(x, \sqrt{t} / 2) \times(3 t / 4, t)$, we obtain

$$
u(x, t)^{2} \leq \frac{C}{\left|Q_{\sqrt{t} / 2}(x, t)\right|} \int_{3 t / 4}^{t} \int_{B(x, \sqrt{t} / 2)} u^{2} .
$$

It follows that

$$
\begin{aligned}
e^{2 \lambda \psi(x)} u(x, t)^{2} & \leq e^{2 \lambda \psi(x)} \frac{C}{\left|Q_{\sqrt{t} / 2}(x, t)\right|} \int_{3 t / 4}^{t} \int_{B(x, \sqrt{t} / 2)} u^{2} \\
& =\frac{C}{\left|Q_{\sqrt{t} / 2}(x, t)\right|} \int_{3 t / 4}^{t} \int_{B(x, \sqrt{t} / 2)} e^{2 \lambda(\psi(x)-\psi(z))} q^{2} \\
& \leq e^{2 \lambda \sqrt{t}} \frac{C}{|B(x, \sqrt{t})|} e^{c_{0} \lambda^{2} t}\|h\|_{2}^{2}
\end{aligned}
$$

Taking the supremum over all $h \in L^{2}(B(y, \sqrt{t}))$ with $\|h\|=1$, we find that

$$
e^{2 \lambda(\psi(x)-\psi(y))} \int_{B(y, \sqrt{t} / 2)} G_{1}(x, t ; z, 0)^{2} d z \leq C e^{4 \lambda \sqrt{t}+c_{0} \lambda^{2} t} \frac{1}{|B(x, \sqrt{t})|} .
$$


Using the mean-value inequality on the second entries of the heat kernel $G_{1}$ backward in time in the cube $B(y, \sqrt{t} / 2) \times[0, t / 4]$, we have

$$
\begin{aligned}
G_{1}(x, t ; y, 0)^{2} & \leq \frac{C}{\left|Q_{\sqrt{t} / 2}(y, t)\right|} \int_{0}^{t / 4} \int_{B(y, \sqrt{t} / 2)} G_{1}(x, t ; z, s)^{2} d z d s \\
& \leq \frac{C}{|B(x, \sqrt{t})||B(y, \sqrt{t})|} \exp \left(4 \lambda \sqrt{t}+c_{0} \lambda^{2} t-2 \lambda(\psi(x)-\psi(y))\right)
\end{aligned}
$$

Here we remark that the second entries of $G_{1}$ satisfies the conjugate equation of $L_{1} u-u_{s}=0$. That is, if $v(z, s)=G_{1}(x, t ; z, s)$, then

$$
\Delta v-2 \nabla f \nabla v-2 \Delta f v-10 F v+v_{s}=0 .
$$

Recall that $\Delta f=-F$. Hence $v$ satisfies

$$
L_{3} v+v_{s}=\Delta v-2 \nabla f \nabla v-8 F v+v_{s}=0 .
$$

From Section 2.3 it is clear that the mean-value inequality still holds on the backward parabolic cube.

Choosing $\lambda=d(x, y) /\left(c_{0} t\right)$ and $\psi$ such that $\psi(x)-\psi(y)=d(x, y)$, we reach

$$
G_{1}(x, t ; y, 0)^{2} \leq \frac{C}{|B(x, \sqrt{t})||B(y, \sqrt{t})|} \exp \left(-\frac{d(x, y)^{2}}{2 c_{0} t}\right) .
$$

This proves the Gaussian upper bound for $G_{1}$.

2.5. A Gaussian upper bound of $G_{2}$ by perturbation. Using the bound for $G_{1}$ and a perturbation argument in [Zhang 1997], we will prove a Gaussian upper bound for $G_{2}$ when $N(V)$ is small.

By Duhamel's formula,

$G_{2}(x, t ; y, 0)=G_{1}(x, t ; y, 0)+10 n \int_{0}^{t} \int_{M} G_{1}(x, t ; z, \tau) V(z) G_{2}(z, \tau ; y, 0) d z d \tau$.

From Section 2.4 we know that, for some $c_{1}, C_{1}>0$,

$$
G_{1}(x, t ; z, \tau) \leq \frac{C_{1}}{|B(x, \sqrt{t-\tau})|} \exp \left(-\frac{c_{1} d(x, z)^{2}}{t-\tau}\right) .
$$

Also, by standard perturbation argument (since $V$ is bounded), there is $M_{\tau}>0$ such that

$$
G_{2}(z, \tau ; y, 0) \leq \frac{M_{\tau}}{|B(z, \sqrt{\tau})|} \exp \left(-\frac{c_{1} d(z, y)^{2}}{\tau}\right) \leq \frac{M_{\tau}}{|B(z, \sqrt{\tau})|} \exp \left(-\frac{c_{1} d(z, y)^{2}}{2 \tau}\right) .
$$

We need to prove that $M_{\tau}$ can be chosen independent of time. 


\section{Therefore}

$$
\begin{aligned}
& G_{2}(x, t ; y, 0) \\
& \quad \leq \frac{C_{1} e^{-c_{1} d(x, y)^{2} / t}}{|B(x, \sqrt{t})|}+C_{1} \int_{0}^{t} \int_{M} \frac{e^{-c_{1} d(x, z)^{2} /(t-\tau)}}{|B(x, \sqrt{t-\tau})|} V(z) \frac{M_{\tau} e^{-c_{1} d(z, y)^{2} /(2 \tau)}}{|B(z, \sqrt{\tau})|} d z d \tau .
\end{aligned}
$$

Let $m_{t}$ be the minimum of the constants $M_{\tau}$ such that

$$
G_{2}(z, \tau ; y, 0) \leq \frac{m_{\tau}}{|B(z, \sqrt{\tau})|} e^{-c_{1} d(z, y)^{2} /(2 \tau)}
$$

for all $\tau \in(0, t]$ and $y, z \in M$. Then

$G_{2}(x, t ; y, 0)$

$$
\leq \frac{C_{1} e^{-c_{1} d(x, y)^{2} / t}}{|B(x, \sqrt{t})|}+C_{1} m_{t} \int_{0}^{t} \int_{M} \frac{e^{-c_{1} d(x, z)^{2} /(t-\tau)}}{|B(x, \sqrt{t-\tau})|} V(z) \frac{e^{-c_{1} d(z, y)^{2} /(2 \tau)}}{|B(z, \sqrt{s})|} d z d \tau .
$$

By [Zhang 1997, Lemma 4.1, p. 1003], there exists a constant $c_{5}$, depending only on the doubling constant $v$, such that

$$
\begin{aligned}
\int_{0}^{t} \int_{M} \frac{e^{-c_{1} d(x, z)^{2} /(t-\tau)}}{|B(x, \sqrt{t-\tau})|} V(z) \frac{e^{-c_{1} d(z, y)^{2} /(2 \tau)}}{|B(z, \sqrt{\tau})|} d z & d \tau \\
& \leq c_{5} M(V) \frac{1}{|B(x, \sqrt{t})|} e^{-c_{1} d(x, y)^{2} /(2 t),},
\end{aligned}
$$

where

$$
M(V) \equiv \sup _{x \in M} \int_{0}^{\infty} \int_{M} \frac{e^{-c_{1} d(x, z)^{2} /(2 t)}}{|B(x, \sqrt{t})|} V(z) d z d t .
$$

In the lemma quoted above, the constant in the exponential term of $M(V)$ was not given explicitly. However, by tracking the proof, one immediately concludes that the coefficient $-c_{1} / 2$ above works. We caution that it is not clear that one can choose the original constant $-c_{1}$, except in the Euclidean case. We mention that the bound above for the integral is the parabolic counterpart of the basic inequality, for $n \geq 3$,

$$
\int_{\mathbb{R}^{n}} \frac{1}{|x-z|^{n-2}}|V(z)| \frac{1}{|z-y|^{n-2}} d z \leq C \sup _{w} \int_{\mathbb{R}^{n}} \frac{|V(y)|}{|y-w|^{n-2}} \frac{1}{|x-y|^{n-2}} d y,
$$

which can be found in many places, including, for instance, [Simon 1982].

Scaling the time variable suitably and use the volume doubling property, we see that

$$
M(V) \leq c N(V)=c \sup _{x \in M} \int_{0}^{\infty} \int_{M} \frac{e^{-d(x, z)^{2} / t}}{|B(x, \sqrt{t})|} V(z) d z d t
$$


Hence

$$
G_{2}(x, t ; y, 0) \leq \frac{C_{1} e^{-c_{1} d(x, y)^{2} / t}}{|B(x, \sqrt{t})|}+C_{1} c_{5} m_{t} N(V) \frac{1}{|B(x, \sqrt{t})|} e^{-c_{1} d(x, y)^{2} /(2 t)} .
$$

Now it follows that

$$
G_{2}(x, t ; y, 0) \leq\left(C_{1}+C_{1} c_{5} m_{t} N(V)\right) \frac{1}{|B(x, \sqrt{t})|} e^{-c_{1} d(x, y)^{2} /(2 t)} .
$$

By the definition of $m_{t}$ we have $m_{t} \leq C_{1}+C_{1} c_{5} m_{t} N(V)$. Hence, if $N(V)<$ $1 /\left(C_{1} c_{5}\right)$, then

$$
m_{t} \leq \frac{C_{1}}{1-C_{1} c_{5} N(V)},
$$

for all $t>0$. Therefore

$$
G_{2}(x, t ; y, 0) \leq \frac{C_{1}}{1-C_{1} c_{5} N(V)} \frac{1}{|B(z, \sqrt{\tau})|} e^{-c_{1} d(x, y)^{2} /(2 t)}
$$

This proves the global upper bound for $G_{2}(x, t ; y, 0)$.

2.6. An $\boldsymbol{L}^{2}$ estimate of $\boldsymbol{F}=|\nabla \log \boldsymbol{u}|^{2}$. Let $\phi=\phi(x)$ be a smooth cutoff function defined in $B(x, 2 r)$ such that $0 \leq \phi \leq 1, \phi(y)=1$ in $B(x, r)$ and $\phi(y)=0$ in $B(x, 2 r)^{c}$. Then

$$
\begin{aligned}
\int_{B(x, 2 r)} F \phi^{2} d y & =\int_{B(x, 2 r)} \frac{\nabla u \nabla u}{u^{2}} \phi^{2} d y=-\int_{B(x, 2 r)} u \operatorname{div}\left(\frac{\nabla u}{u^{2}} \phi^{2}\right) d y \\
& =-\int_{B(x, 2 r)} u \frac{\Delta u}{u^{2}} \phi^{2} d y-\int_{B(x, 2 r)} u \nabla u \nabla\left(\frac{\phi^{2}}{u^{2}}\right) d y \\
& =2 \int_{B(x, 2 r)} \frac{u \nabla u \nabla u}{u^{3}} \phi^{2} d y-2 \int_{B(x, 2 r)} u \nabla u \frac{\phi \nabla \phi}{u^{2}} d y \\
& =2 \int_{B(x, 2 r)} F \phi^{2} d y-2 \int_{B(x, 2 r)} u \nabla u \frac{\phi \nabla \phi}{u^{2}} d y .
\end{aligned}
$$

Therefore

$$
\int_{B(x, 2 r)} \frac{|\nabla u|^{2}}{u^{2}} \phi^{2} d y \leq 2 \int_{B(x, 2 r)} \frac{|\nabla u|}{u} \phi|\nabla \phi| d y .
$$

Hence

$$
\int_{B(x, 2 r)} \frac{|\nabla u|^{2}}{u^{2}} \phi^{2} d y \leq 4 \int_{B(x, 2 r)}|\nabla \phi|^{2} d y .
$$

This implies that

$$
\int_{B(x, r)} F(y) d y=\int_{B(x, r)} \frac{|\nabla u|^{2}}{u^{2}} d y \leq 4 \frac{|B(x, 2 r)|}{r^{2}} .
$$


2.7. A mean-value inequality for solutions of $\boldsymbol{L}_{\mathbf{2}} \boldsymbol{w}-\boldsymbol{w}_{\boldsymbol{t}} \geq \mathbf{0}$. (See (2-2) for the definition of $L_{2}$.)

Let $\psi$ be the smooth cutoff function defined on page 385, with $\sigma=2$. Take $r$ there to be $r / 2$. Since $\psi$ is supported in $Q_{r}(x, t)$, we know that

$$
\begin{aligned}
\Delta(w \psi)+2 \nabla f \nabla(w \psi)-10 F(w \psi) & +10 n V(w \psi)-(w \psi)_{t} \\
\geq & 2(\nabla f \nabla \psi) w+(\Delta \psi) w-w \psi_{t}+2 \nabla \psi \nabla w .
\end{aligned}
$$

Since $G_{2}$ is the fundamental solution of the left-hand side, we have

$$
\begin{aligned}
w(x, t) \leq-2 \int_{Q_{r}(x, t)} G_{2}(x, t ; y, s) & (\nabla f \nabla \psi) w d y d s \\
& -\int_{Q_{r}(x, t)} G_{2}(x, t ; y, s)\left((\Delta \psi) w-w \psi_{t}\right) d y d s \\
& -\int_{Q_{r}(x, t)} G_{2}(x, t ; y, s) 2 \nabla \psi \nabla w d y d s .
\end{aligned}
$$

After integration by parts, this becomes, setting $v=v(y, s)=G_{2}(x, t ; y, s)$ :

$$
\begin{gathered}
w(x, t) \leq-2 \int_{Q_{r}(x, t)} v \nabla f \nabla \psi w d y d s+\int_{Q_{r}(x, t)} v w \psi_{t} d y d s \\
\quad+\int_{Q_{r}(x, t)} \nabla_{y} v \nabla \psi w d y d s-\int_{Q_{r}(x, t)} v \nabla \psi \nabla w d y d s \\
\equiv J_{1}+J_{2}+J_{3}+J_{4} .
\end{gathered}
$$

We estimate each integral separately.

Since $(x, t)$ is bounded away from the supports of the functions $\nabla \psi$ and $\psi_{t}$ by a parabolic distance of $r$, we have

$$
v \leq \frac{C}{|B(x, r)|}
$$

in all the integrals on the right-hand side of (2-15). Hence (2-15) implies

$$
\begin{aligned}
\left|J_{1}+J_{2}\right| \leq \frac{C}{|B(x, r)|}\left(\int_{Q_{r}(x, t)}|\nabla f|^{2} d y d s\right)^{1 / 2}\left(\int_{Q_{r}(x, t)} w^{2} d y d s\right)^{1 / 2} & \\
& +\frac{C}{r^{2}|B(x, r)|} \int_{Q_{r}(x, t)} w d y d s .
\end{aligned}
$$

Using $|\nabla f|^{2}=F=|\nabla u|^{2} / u^{2}$, by (2-14), we deduce

$$
\begin{aligned}
& w(x, t) \\
& \leq\left(\frac{C}{r^{2}|B(x, r)|} \int_{Q_{r}(x, t)} w^{2} d y d s\right)^{1 / 2}+\frac{C}{r^{2}|B(x, r)|} \int_{Q_{r}(x, t)} w d y d s+\left|J_{3}\right|+\left|J_{4}\right| .
\end{aligned}
$$


This shows, by Hölder's inequality,

$$
w(x, t) \leq\left(\frac{C}{r^{2}|B(x, r)|} \int_{Q_{r}(x, t)} w^{2} d y d s\right)^{1 / 2}+\left|J_{3}\right|+\left|J_{4}\right| .
$$

In the next two steps we will find a bound for the last integrals in (2-17).

2.8. Controlling the remaining integrals. There remains to control the terms

$$
J_{3}=\int_{Q_{r}(x, t)} \nabla_{y} v \nabla \psi w d y d s
$$

and

$$
J_{4}=\int_{Q_{r}(x, t)} v \nabla \psi \nabla w d y d s
$$

We deal with $J_{3}$ first. Using (2-16) and Hölder's inequality, we get

$$
\left|J_{3}\right| \leq \frac{C}{r}\left(\int_{Q_{r}(x, t)-Q_{r / 2}(x, t)}\left|\nabla_{y} v\right|^{2} d y d s\right)^{1 / 2}\left(\int_{Q_{r}(x, t)} w^{2} d y d s\right)^{1 / 2} .
$$

What remains is to estimate the first factor on the right-hand side.

Since $G_{2}$ is the heat kernel of $L_{2}$, in other words the fundamental solution of the operator

$$
\Delta+2 \nabla f \nabla-10 F+10 n V-\partial_{s},
$$

we know that $v=G_{2}(x, t ; y, s)$ is a solution of the conjugate of $L_{2}-\partial_{s}$, except at $(x, t)$ :

$$
\Delta v-2 \nabla f \nabla v-2 \Delta f v-10 F v+10 n V v+v_{s}=0 .
$$

Since $\Delta f=-F$, this becomes

$$
\Delta v-2 \nabla f \nabla v-8 F v+10 n V v+v_{s}=0
$$

that is, $L_{3} v+v_{s}+10 n V v=0$.

Take a suitable cutoff function $\psi_{1}$ and use $\psi_{1}^{2} v$ as a test function on (2-20) and $h=10 n V v$ in (2-8). We can follow the argument between (2-3) and (2-8) verbatim to obtain

$$
\begin{aligned}
\int_{Q_{r}(x, t)-Q_{r / 2}(x, t)} & \left|\nabla_{y} v\right|^{2} d y d s \\
\leq & \frac{C}{r^{2}} \int_{Q_{2 r}(x, t)-Q_{r / 4}(x, t)} v^{2} d y d s+10 n \int_{Q_{4 r}(x, t)-Q_{r / 4}(x, t)} V v^{2} d y d s .
\end{aligned}
$$


It follows that (recalling that $v=G_{2}(x, t ; y, s)$ )

$$
\begin{aligned}
\int_{Q_{r}(x, t)-Q_{r / 2}(x, t)}\left|\nabla_{y} v\right|^{2} d y d s \leq \frac{C}{r^{2}} \int_{Q_{2 r}(x, t)-Q_{r / 4}(x, t)} v^{2} d y d s & \\
+10 n \sup _{\substack{(y, s) \in \\
Q_{4 r}(x, t)-Q_{r / 4}(x, t)}} G_{2}(x, t ; y, s) & \int_{Q_{4 r}(x, t)-Q_{r / 4}(x, t)} V v d y d s .
\end{aligned}
$$

Using the Gaussian bound on $G_{2}$ and the assumption on $V$ we get

$$
\int_{Q_{r}(x, t)-Q_{r / 2}(x, t)}\left|\nabla_{y} G_{2}(x, t ; y, s)\right|^{2} d y d s \leq C \frac{1+N(V)}{|B(x, r)|},
$$

where we have used the inequality

$$
\int_{Q_{4 r}(x, t)-Q_{r / 4}(x, t)} V G_{2}(x, t ; y, s) d y d s \leq N(V),
$$

which comes from the Gaussian upper bound of $G_{2}$ and rescaling in time; see Assumption C. Inserting the $L^{2}$ estimate for the gradient of $G_{2}$ in (2-19) we obtain

$$
\left|J_{3}\right| \leq\left(\frac{C}{r^{2}|B(x, r)|} \int_{Q_{4 r}(x, t)} w^{2} d y d s\right)^{1 / 2} .
$$

Next we turn our attention to the last remaining integral, defined in (2-18). Using (2-16) and Hölder's inequality we reach

$$
\begin{aligned}
\left|J_{4}\right| & \leq \frac{C}{r}\left(\int_{Q_{r}(x, t)-Q_{r / 2}(x, t)} G_{2}(x, t ; y, s)^{2} d y d s\right)^{1 / 2}\left(\int_{Q_{r}(x, t)}|\nabla w|^{2} d y d s\right)^{1 / 2} \\
& \leq C\left(\frac{1}{|B(x, r)|} \int_{Q_{r}(x, t)}|\nabla w|^{2} d y d s\right)^{1 / 2} .
\end{aligned}
$$

Recall that $L_{2} w-w_{s} \geq 0$, and hence

$$
L_{3} w-w_{s}+10 n V w \geq 0
$$

where $L_{3}$ is defined at the start of Section 2.3.

Taking $h=10 n V w$ and $\sigma=2$ in (2-8), we obtain

$$
\int_{Q_{2 r}(x, t)}\left|\nabla\left(\psi_{2} w\right)\right|^{2} d y d s \leq \frac{C}{r^{2}} \int_{Q_{2 r}} w^{2} d y d s+10 n \int_{Q_{\sigma r}} V\left(w \psi_{2}\right)^{2} d y d s .
$$


Here $\psi_{2}$ is the cutoff function in (2-8) with $\sigma=2$. By Assumption $\mathrm{C}$ for $V$ we have, for a constant $C^{\prime}$,

$$
\int_{Q_{r}(x, t)}|\nabla w|^{2} d y d s \leq \int_{Q_{2 r}(x, t)}\left|\nabla\left(\psi_{2} w\right)\right|^{2} d y d s \leq \frac{C^{\prime}}{r^{2}} \int_{Q_{2 r}} w^{2} d y d s .
$$

From this and (2-22), we get

$$
J_{4} \leq C\left(\frac{1}{r^{2}|B(x, r)|} \int_{Q_{2 r}(x, t)} w^{2} d y d s\right)^{1 / 2} .
$$

Substituting this and (2-21) to (2-17), we reach

$$
w(x, t) \leq\left(\frac{C}{r^{2}|B(x, r)|} \int_{Q_{4 r}(x, t)} w^{2} d y d s\right)^{1 / 2} .
$$

2.9. Completion of the proof. Recall that $w=F^{5 n}$ and that $w$ is independent of time. Hence (2-21) becomes

$$
F(x) \leq\left(\frac{C}{|B(x, r)|} \int_{B(x, 2 r)} F^{10 n} d y\right)^{1 /(10 n)}
$$

By a modification of a well known trick [Li and Schoen 1984], this inequality implies

$$
F(x) \leq \frac{C}{|B(x, r)|} \int_{B(x, 2 r)} F d y
$$

(In that paper it was shown that an $L^{2}$ mean-value inequality implies an $L^{1}$ meanvalue inequality. Applying the same method, one can deduce (2-25) from (2-24) without difficulty.)

Combining (2-25) with (2-14), we have

$$
\frac{|\nabla u|^{2}}{u^{2}}=F(x) \leq \frac{C}{|B(x, r)|} \int_{B(x, 2 r)} F d y \leq \frac{C}{r^{2}} .
$$

This finishes the proof of the global gradient bound, and of the theorem.

\section{Acknowledgment}

I thank Professors Thierry Coulhon and Peter Li for helpful suggestions.

\section{References}

[Ancona 1990] A. Ancona, "Théorie du potentiel sur les graphes et les variétés", pp. 1-112 in École d'été de Probabilités de Saint-Flour XVIII (1988), edited by P. L. Hennequin, Lecture Notes in Math. 1427, Springer, Berlin, 1990. MR 92g:31012 Zbl 0719.60074 
[Carron 1997] G. Carron, "Inégalités de Hardy sur les variétés riemanniennes non-compactes", $J$. Math. Pures Appl. (9) 76:10 (1997), 883-891. MR 99c:53026 Zbl 0886.58111

[Cheng and Yau 1975] S. Y. Cheng and S. T. Yau, "Differential equations on Riemannian manifolds and their geometric applications", Comm. Pure Appl. Math. 28:3 (1975), 333-354. MR 52 \#6608 Zbl 0312.53031

[Coulhon and Saloff-Coste 1995] T. Coulhon and L. Saloff-Coste, "Variétés riemanniennes isométriques à l'infini”, Rev. Mat. Iberoamericana 11 (1995), 687-726. MR 96m:53035 Zbl 0845.58054

[Davies 1989] E. B. Davies, Heat kernels and spectral theory, Cambridge Tracts in Mathematics 92 , Cambridge University Press, Cambridge, 1989. MR 90e:35123 Zbl 0699.35006

[Grigoryan 1991] A. A. Grigoryan, "The heat equation on noncompact Riemannian manifolds", Mat. Sbornik 182:1 (1991), 55-87. In Russian; translated in Math. USSR Sb. 72 (1992), 47-77. MR 92h:58189 Zbl 0776.58035

[Grigor'yan 1999] A. Grigor'yan, "Estimates of heat kernels on Riemannian manifolds", pp. 140225 in Spectral theory and geometry (Edinburgh, 1998), edited by B. Davies and Y. Safarov, London Math. Soc. Lecture Note Ser. 273, Cambridge Univ. Press, Cambridge, 1999. MR 2001b:58040 Zbl 0985.58007

[Li and Schoen 1984] P. Li and R. Schoen, " $L^{p}$ and mean value properties of subharmonic functions on Riemannian manifolds", Acta Math. 153:3-4 (1984), 279-301. MR 86j:58147 Zbl 0556.31005

[Li and Wang 1999] P. Li and J. Wang, "Mean value inequalities", Indiana Univ. Math. J. 48:4 (1999), 1257-1283. MR 2001e:58032 Zbl 1003.58026

[Li and Yau 1986] P. Li and S.-T. Yau, "On the parabolic kernel of the Schrödinger operator", Acta Math. 156:3-4 (1986), 153-201. MR 87f:58156 Zbl 0940.35205

[Saloff-Coste 1992] L. Saloff-Coste, "A note on Poincaré, Sobolev, and Harnack inequalities", Internat. Math. Res. Notices 2 (1992), 27-38. MR 93d:58158 Zbl 0769.58054

[Saloff-Coste 2002] L. Saloff-Coste, Aspects of Sobolev-type inequalities, London Math. Soc. Lecture Note Ser. 289, Cambridge Univ. Press, Cambridge, 2002. MR 2003c:46048 Zbl 0991.35002

[Simon 1982] B. Simon, "Schrödinger semigroups", Bull. Amer. Math. Soc. (N.S.) 7:3 (1982), 447526. MR 86b:81001a Zbl 0524.35002

[Yau 1975] S. T. Yau, "Harmonic functions on complete Riemannian manifolds", Comm. Pure Appl. Math. 28 (1975), 201-228. MR 55 \#4042 Zbl 0291.31002

[Zhang 1997] Q. S. Zhang, "On a parabolic equation with a singular lower order term, II: The Gaussian bounds”, Indiana Univ. Math. J. 46:3 (1997), 989-1020. MR 98m:35079 Zbl 0909.35054

Received September 9, 2004.

QI S. ZHANG

DEPARTMENT OF MATHEMATICS

UNIVERSITY OF CALIFORNIA

RIVERSIDE, CA 92521

UNITED STATES

qizhang@math.ucr.edu 